\title{
Iterative dummy area method with flexible dummy area size for the design of kinoform
}

\author{
Toshinori Hora ${ }^{\mathrm{a},}$, Shiyuan Yang ${ }^{\mathrm{a}}$ \\ ${ }^{a}$ Kyushu Institute of Technology \\ 1-1 Sensui-cho, Tobata-ku, Kitakyushu, Fukuoka, JAPAN 804-8550 \\ *Corresponding Author. hora@boss.ecs.kyutech.ac.jp
}

\begin{abstract}
As an algorithm that generates a kinoform is a phase-type computer-generated hologram, there is a iterative dummy area method. However, it is to reduce the error in the original image space, using the degree of freedom in the dummy area, the dummy area method is repeated dummy area error occurs. That the error occurs in the dummy region, undesirable in terms of light use efficiency. In this study, we aim to reduce the error of the dummy area there. In the computer generated hologram, the reconstructed image is modulated by a sinc function in both the horizontal direction, vertical direction. Therefore, compared to the reconstructed image is near the center, the periphery will be dark. In the computer generated hologram, the reconstructed image is modulated by a sinc function in both the horizontal direction, vertical direction. First, instead of using the whole dummy area Thus, by going to increase the dummy area is used gradually, it is possible to concentrate on the periphery error leads to a reduction in error.
\end{abstract}

Keywords : Kinoform, iterative dummy area method.

\section{Introduction}

Kinoform is a hologram that records only the phase information of the object wave. High utilization efficiency of light from the unnecessary diffracted wave does not occur during reproduction, the kinoform, the principle use efficiency of 100 [\%] is possible. Further, the kinoform to play two-dimensional image reproduction because it is the intensity, I can optionally be given the phase of the original image. Therefore, there is a remarkable that the distribution of spike appears often requires extremely wide dynamic

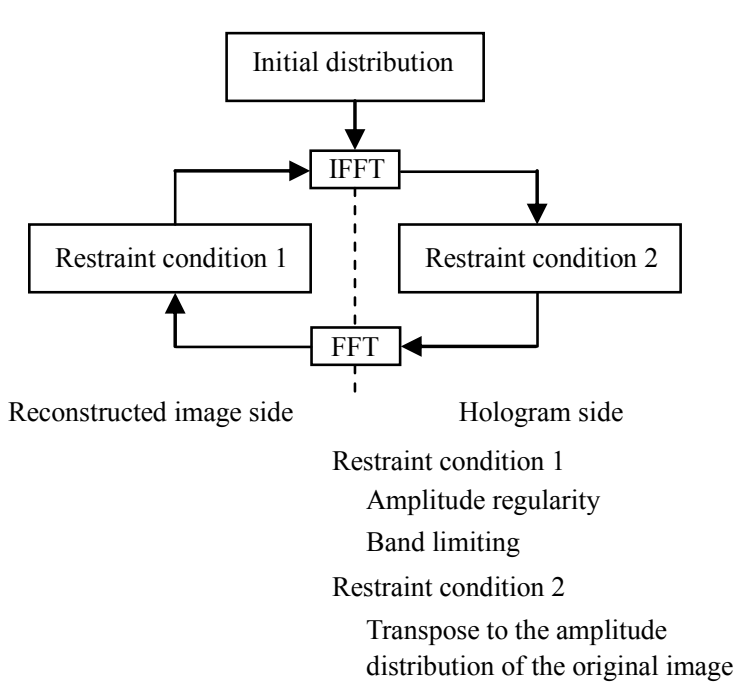

Fig. 1. Iterative dummy area method

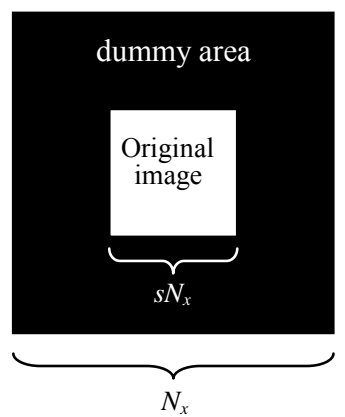

Fig. 2. Dummy area

range and performing a Fourier transform, using a random phase to the phase of the reconstructed image.

In addition, we must constant amplitude distribution for in kinoform does not record the amplitude information. By replacing the constant amplitude distribution, error occurs in the reproduced image I, however. As an algorithm to reduce the error in this case, the dummy area method is used repeatedly. 


\section{Iterative dummy area method}

Shows the flow of repeated dummy domain method in Fig. 1. Add a dummy area around the image of the initial value of zero to the original method, the dummy area repeatedly, the input image and the image. By using the degree of freedom of the amplitude and phase of the dummy region, the error can be reduced.

And the size of the city when added to the original image, the dummy area the size of the original image. Figure 2 shows the image after the addition. Initial input signal when the original image and the initial distribution of the

$$
\begin{aligned}
g_{0}(x, y)= & \left|g_{0}(x, y)\right| \exp \{j \varphi(x, y)\} \\
= & \left\{\begin{array}{l}
|f(x, y)| \exp \left\{j \varphi_{0}(x, y)\right\} \\
:-\frac{s N_{x}}{2} \leq x \leq \frac{s N_{x}}{2}-1,-\frac{s N_{y}}{2} \leq y \leq \frac{s N_{y}}{2}-1 \\
0 \\
: \text { otherwise }
\end{array}\right.
\end{aligned}
$$

However, $\varphi(x, y)$ is the random phase. When $G_{0}(u, v)$ and the inverse Fourier transform of equation (1)

$$
\begin{aligned}
G_{0}(u, v)= & \frac{1}{\sqrt{N_{x} N_{y}}} \\
& \quad \times \cdot \sum \sum g_{0}(x, y) \exp \left\{j 2 \pi\left(\frac{u x}{N_{x}}+\frac{v y}{N_{y}}\right)\right\} \\
= & \left|G_{0}(u, v)\right| \exp \left\{j \phi_{0}(u, v)\right\}
\end{aligned}
$$

As constraints in the hologram plane, then make a constant amplitude and bandwidth limitation. When the A signal and replaced after a certain value $G_{0}^{\prime}(u, v)$ width was subjected to constraints

$$
G_{0}^{\prime}(u, v)=\left\{\begin{array}{l}
A \exp \left\{j \phi_{0}(u, v)\right\} \\
:-\frac{t N_{x}}{2} \leq x \leq \frac{t N_{x}}{2}-1,-\frac{t N_{y}}{2} \leq y \leq \frac{t N_{y}}{2}-1 \\
0 \\
: \text { otherwise }
\end{array}\right.
$$

By Fourier transform obtained wherein the $G_{0}^{\prime}(u, v)$, $g_{0}^{\prime}(x, y)$ reproduction image can be obtained.

$$
g_{0}^{\prime}(x, y)=\left|g_{0}^{\prime}(x, y)\right| \exp \left\{j \varphi_{0}^{\prime}(x, y)\right\}
$$

In addition, we substitute the following as constraints in terms of reconstructed image. The replacement signal as $g_{1}(x, y)$

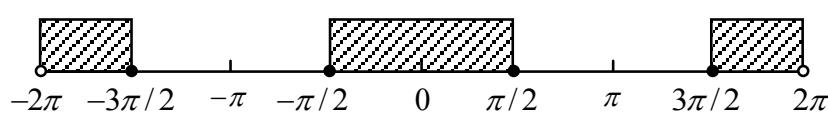

Fig. 3. Phase difference restrictions

$$
g_{1}(x, y)=\left\{\begin{array}{l}
|f(x, y)| \exp \left\{j \varphi_{0}^{\prime}(x, y)\right\} \\
:-\frac{s N_{x}}{2} \leq x \leq \frac{s N_{x}}{2}-1,-\frac{s N_{y}}{2} \leq y \leq \frac{s N_{y}}{2}-1 \\
a_{0} g_{0}^{\prime}(x, y) \\
: \text { otherwise }
\end{array}\right.
$$

but

$$
a_{0}=\frac{\sum \sum|f(x, y)|^{2}\left|g_{0}^{\prime}(x, y)\right|^{2}}{\sum \sum\left|g_{0}^{\prime}(x, y)\right|^{4}}
$$

It is a new input and the equation (5), we will repeat the above procedure is repeated dummy domain method. Input at $\mathrm{k}+1$ th iteration Assuming $g_{k+1}(x, y)$

$$
g_{k+1}(x, y)=\left\{\begin{array}{l}
|f(x, y)| \exp \left\{j \varphi_{k}^{\prime}(x, y)\right\} \\
:-\frac{s N_{x}}{2} \leq x \leq \frac{s N_{x}}{2}-1,-\frac{s N_{y}}{2} \leq y \leq \frac{s N_{y}}{2}-1 \\
a_{k} g_{k}^{\prime}(x, y) \\
: \text { otherwise }
\end{array}\right.
$$

$$
a_{k}=\frac{\sum \sum|f(x, y)|^{2}\left|g_{k}^{\prime}(x, y)\right|^{2}}{\sum \sum\left|g_{k}^{\prime}(x, y)\right|^{4}}
$$

However, the speckle will occur from the fact that the reproduction image by using a random phase. And speckle occurs because of noise that appears in the hologram technology in general, is used coherent light such as laser light of the hologram recording playback. To give a large influence for the reproduction image, speckle need to remove speckle. In order to remove the speckle Therefore, to limit the phase difference in the range shown in Figure 3. Here, the phase difference at each point in the reconstructed image and the phase difference. In this study, the phase difference method is referred to as the Act on Limitation of this speckle removal. In addition to the constraints in terms of the reconstructed image domain method dummy Repeat this phase difference limit law.

\section{Iterative variable dummy area method}

However, the speckle will occur from the fact that the reproduction image by using a random phase. And speckle occurs because of noise that appears in the hologram technology in general, is used coherent light such as laser light of the hologram

From Figure 4, it can be seen that the error in the dummy region have come up a lot. Considering the optical reproduction is undesirable in terms of light use efficiency 


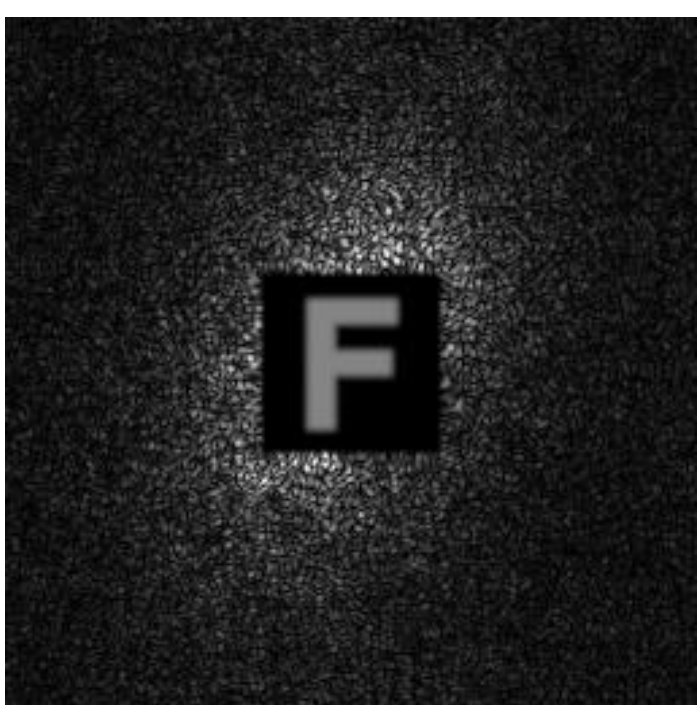

Fig. 4. Result

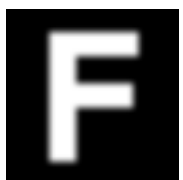

Fig. 5. Original image

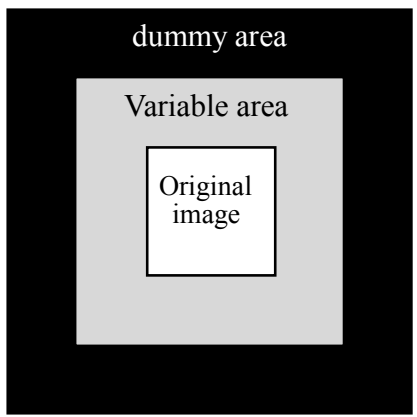

Fig. 6. Variable area

that there is an error in the dummy region. When playing the computer generated hologram Therefore, using the characteristics that are modulated by the sinc function in both the horizontal direction, vertical direction, the peripheral portion is dark.

First, add a dummy area is another variation area around the original image. Original image as a new area including the area variation area and the original image area, the dummy area method is performed repeatedly. Repetition progresses, we gradually reduce the fluctuation region. This fluctuation region, varied in the range of the original image area dummy area, up to a minimum.

\section{Simulation and results}

I was simulated under the following conditions.
- Images

Images with $64 \times 64$ pixel

- Initial phase shown in Fig. 5

- Dummy area

- Band limiting Random phase $256 \times 256[$ pixel] $1 / 2$

- It is repeatedly a frequency 1000 times

Reproduced image error rate is performed by the following equation.

$$
a_{0}=10 \cdot \log \frac{\sum \sum|f(x, y)|^{2}-\alpha\left|g_{k}^{\prime}(x, y)\right|^{2}}{\sum \sum\left|g_{k}^{\prime}(x, y)\right|^{4}}
$$

We shows the results of simulation in Fig7(a),8(a). Fig(b) is taken out in which only the region in the original image in Fig7(a),8(a). Fig7(b),8(b) shows that beyond the maximum luminance value of 255 to a maximum value of the amplitude of the original image area is the result when all replaced with 255 also in Fig7(a),8(a).

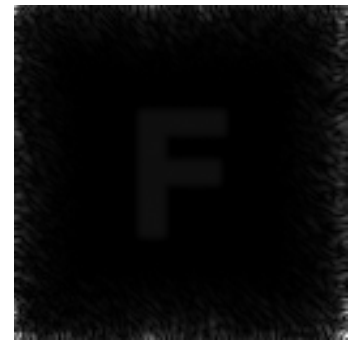

(a) The result including the dummy area

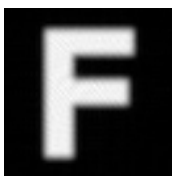

(b) The result of the original image area only

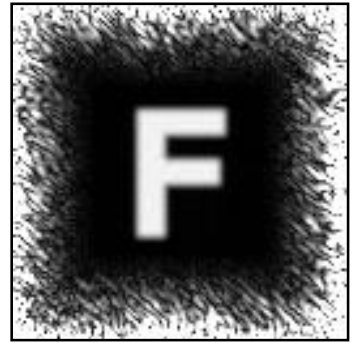

(c) Brightness value is max value in original area

Fig. 7. Result(Dummy area:128) 


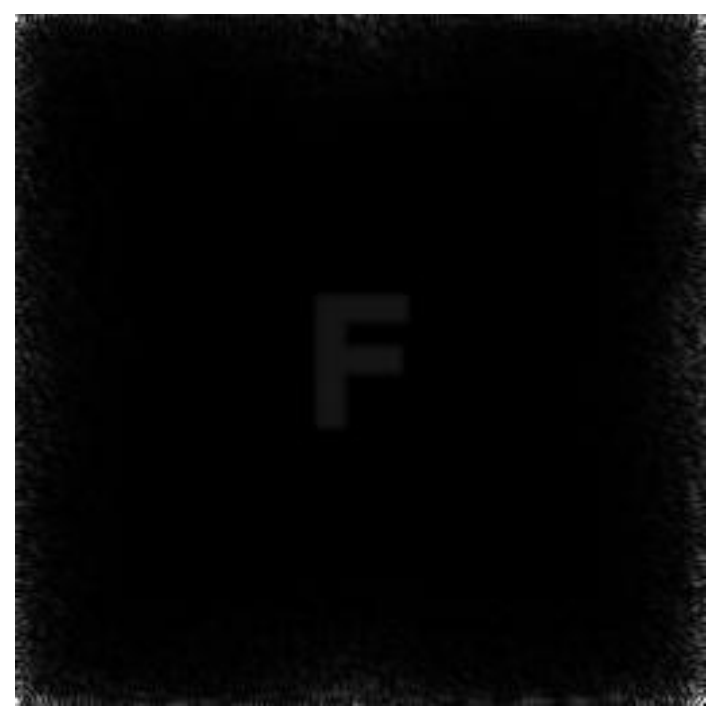

(a) The result including the dummy area

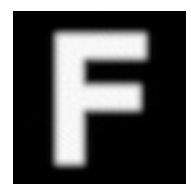

(b) The result of the original image area only

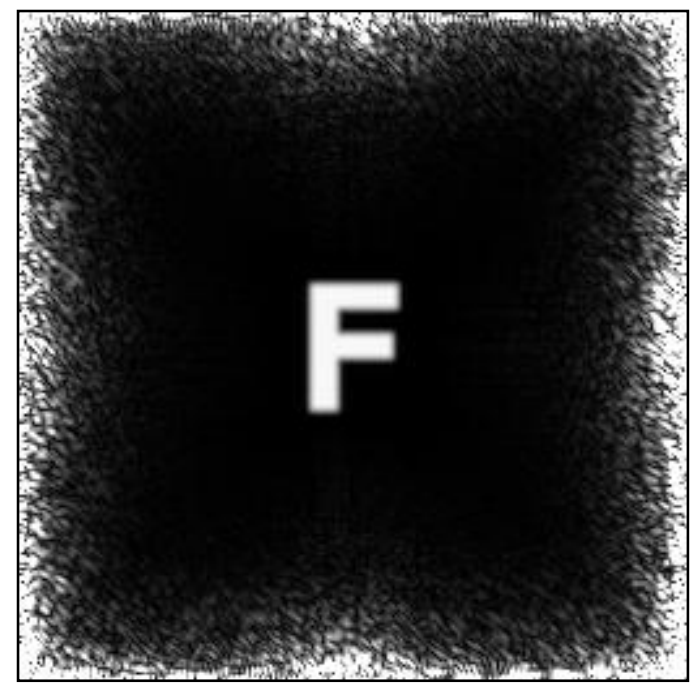

(c) Brightness value is max value in original area

Fig. 8. Result(Dummy area:256)

I find that the error does not occur in the vicinity of substantially the original image area from Figure 7 , but a large error out to the periphery of the reproduced image. Further, this reproduced image error is $-34.5[\mathrm{~dB}]$, that error can be reduced even if the same level of the reproduced image in comparison with the usual method of repeating the dummy region.

\section{Conclusion}

Repeating dummy area method reduces the error of the original image area by using the degree of freedom in the dummy region. Because that would cause an error in the dummy area, it is undesirable in terms of light use efficiency, however. In this study, we were able to reduce the error in the vicinity of the original image by also definitive region where fluctuations. In addition, the reproduced image error, the error can be reduced to about the same as the conventional method of repeated dummy area.

\section{References}

(1) H. Aagedal, M. Schmid, T. Beth, S. Teiwes, and F. Wyrowski, "Theory of speckles in diffractive optics andits application to beam shaping," J. Mod. Opt. No. 43, 1409 - 1421 (1996).

(2) H. Akahori, "Spectrum leveling by an iterative algorithm with a dummy area for synthesizing the kinoform,” Appl. Opt., Vol. 25, No. 5, 802-811 (1986).

(3) R.Braer, F.Wyrowski, and O.Bryungdahl, "Diffusers in digital holography”, J.Opt.Soc.Am.A, Vol. 8 No. 3, 572-578(1991).

(4) F. Wyrowsky and O. Bryngdahl, "Speckle-free reconstruction in digital holography," J. Opt. Soc. Am. A, Vol. 6, No. 8, 1171-1170 (1989).

(5) Shiyuan Yang and Hiroaki Takajo, "Speckle reduction of kinoform reconstruction

(6) Name : "English Title", Journal, Volume, Number, Pages, Year 